\title{
Scattering of terahertz radiation on a graphene-based nano-antenna
}

\author{
Ignacio Llatser*, ${ }^{*}$, Christian Kremers ${ }^{\dagger}$, Albert Cabellos-Aparicio*, Josep Miquel \\ Jornet**, Eduard Alarcón* and Dmitry N. Chigrin ${ }^{\dagger}$ \\ *Nanonetworking Center in Catalunya (N3Cat), Universitat Politècnica de Catalunya, Jordi Girona 1-3, \\ Campus Nord, D6-008 08034 Barcelona, Spain \\ ${ }^{\dagger}$ Theoretical Nano-Photonics Group, Institute of High-Frequency and Communication Technology, \\ Faculty of Electrical, Information and Media Engineering, University of Wuppertal, \\ Rainer-Gruenter-Str. 21, D-42119 Wuppertal, Germany \\ ** Broadband Wireless Networking Laboratory, School of Electrical and Computer Engineering, Georgia \\ Institute of Technology, Atlanta, Georgia 30332, USA
}

\begin{abstract}
Scattering of the terahertz radiation on a graphene-based nano-antenna is considered. Different electromagnetic models of graphene are discussed and applied to calculate extinction, scattering and absorption cross sections of the nanoantenna. Scattering resonances in the terahertz band are identified as longitudinal Fabry-Perot resonances of surface plasmon polaritons supported by the graphene layer. A simple while powerful model, based on the effective mode index of plasmon polaritons, is proposed to predict the antenna resonant properties. A systematic numerical study of the graphene-based nanoantenna is presented for different antenna dimensions. Finally, the potential of graphene-based nano-antennas for terahertz applications is discussed.
\end{abstract}

Keywords: light scattering, nano-antenna, terahertz radiation, graphene

PACS: 78.67.Wj, 84.40.Ba, 73.20.Mf

Graphene, a flat monolayer of carbon atoms tightly packed in a two-dimensional honeycomb lattice, has recently attracted the attention of the research community due to its novel mechanical, thermal, chemical, electronic and optical properties [1]. Graphene has given rise to a plethora of potential applications ranging from ultra high-speed transistors [2] to transparent solar cells [3]. Among these, a particularly promising emerging field is graphene-enabled wireless communications. Indeed, graphene-based nano-antennas [4] just a few micrometers in size are envisaged to radiate electromagnetic waves in the terahertz band, thereby enabling wireless communications among nanodevices [5].

A graphene layer can be modeled as an infinitesimally thin two-sided surface. An associated surface conductivity is calculated within the Kubo formalism and, in the case of the electrostatic bias, one can distinguish between purely interband and intraband contributions [6, 7]. In the terahertz frequency range, the intraband contribution dominates. Within the random-phase approximation, the surface conductivity of graphene can be represented in a local, Drude-like form $[6,7]$ :

$$
\sigma=\frac{2 e^{2}}{\pi \hbar} \frac{k_{B} T}{\hbar} \ln \left[2 \cosh \left[\frac{\mu_{c}}{2 k_{B} T}\right]\right] \frac{i}{\omega+i \tau^{-1}}
$$

where $\tau$ is the total relaxation time of intraband electron scattering processes, $T$ is the temperature and $\mu_{c}$ is the chemical potential. Here, we assume room temperature $(T=300 \mathrm{~K})$, zero electrostatic bias $\left(\mu_{c}=0\right)$ and a conservative value of the relaxation time $\left(\tau=10^{-13} \mathrm{~s}\right)$.

In order to numerically study electromagnetic response of graphene, it is necessary to self-consistently couple the phenomenological conductivity model (1) with the three-dimensional Maxwell's equations. The major complication here is that a typical numerical scheme to solve Maxwell's equations involves finite-size discretization of space, which makes modeling of the infinitesimally thin graphene layer a challenging task. In what follows, we propose and compare two different approaches to solve this problem.

In the first approach, graphene is approximated by a thin, but finite, slab with a tensor effective conductivity

$$
\overleftrightarrow{\sigma}=\left(\begin{array}{ccc}
\sigma / \Delta & 0 & 0 \\
0 & \sigma / \Delta & 0 \\
0 & 0 & 0
\end{array}\right)
$$

where $\Delta$ is the thickness of an equivalent slab and the graphene layer is located in $x-y$ plane. The tensor structure of the conductivity (2) together with the relation $\lim _{\Delta \rightarrow 0} 1 / \Delta=\delta(z)$ ensure that, in the infinitesimally thin slab limit, the induced current is purely two-dimensional. In figure 1 , the method is applied to the problem of terahertz radiation scattering on an $10 \mu \mathrm{m}$ wide graphene 


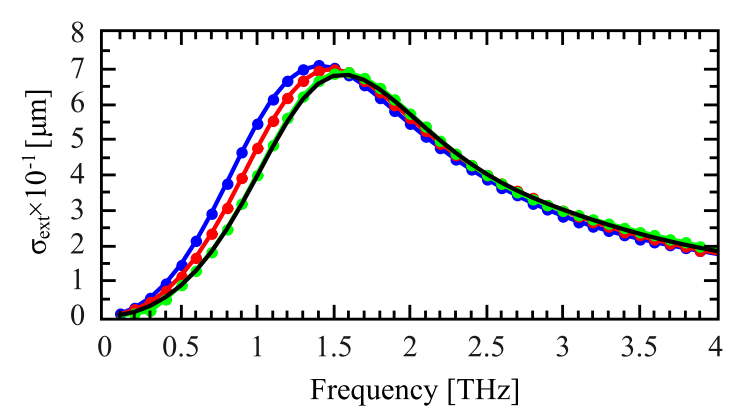

FIGURE 1. Extinction cross section per unit width of an $10 \mu \mathrm{m}$ wide graphene patch with length $L=5 \mu \mathrm{m}$. Results of the impedance surface model (solid black line) and the equivalent slab model (lines with dots) are shown. The thicknesses of the equivalent graphene slab $\Delta$ are $500 \mathrm{~nm}, 200 \mathrm{~nm}$ and $5 \mathrm{~nm}$, from left to right.

patch with length $L=5 \mu \mathrm{m}$. A plane wave at normal incidence, with electric field polarized along the stripe length, is considered. Numerical calculations were done using the method of moments with surface equivalence principle [8]. In figure 1, the extinction cross section of an equivalent graphene slab is shown for different thicknesses: 500, 200 and $5 \mathrm{~nm}$. Results converge both for the spectrum shape and the position of the resonance frequency. The origin of the resonant character of the extinction spectra is discussed below.

The main disadvantage of the proposed method is that one has to model resonant structures with high aspect ratios $(L / \Delta \sim 1000)$, which inevitably leads to high mesh densities and hence high computational costs. To simplify the problem, we propose to model graphene as an equivalent impedance surface. The surface impedance $Z_{S}$ connects the tangential component of the electric field on the surface with the electric surface current, $\left.\mathbf{E}_{\perp}\right|_{z=0}=$

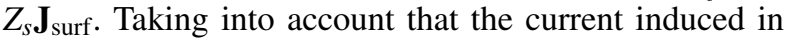
the graphene layer is purely superficial and it is related to the tangential component of the electric field via surface conductivity as $\mathbf{J}_{\text {surf }}=\left.\sigma \mathbf{E}_{\perp}\right|_{z=0}$, one can define the boundary conditions at the graphene interface:

$$
\hat{\mathbf{n}} \times\left[\left.\mathbf{H}\right|_{z=+0}-\left.\mathbf{H}\right|_{z=-0}\right]=\mathbf{J}_{\text {surf }}=\left.\frac{1}{Z_{s}} \mathbf{E}_{\perp}\right|_{z=0},
$$

where $Z_{s}=1 / \sigma$ is the equivalent surface impedance of the graphene. The boundary conditions (3) fully determine the electromagnetic problem. Figure 1 shows the extinction cross section of an $10 \mu \mathrm{m}$ wide graphene patch, modeled as an equivalent impedance surface with surface impedance $Z_{s}=1 / \sigma$. The stripe length is $L=5 \mu \mathrm{m}$. Calculations have been performed using the method of moments with surface equivalence principle [8]. An excellent agreement with the equivalent slab method can be achieved at lower computational costs.
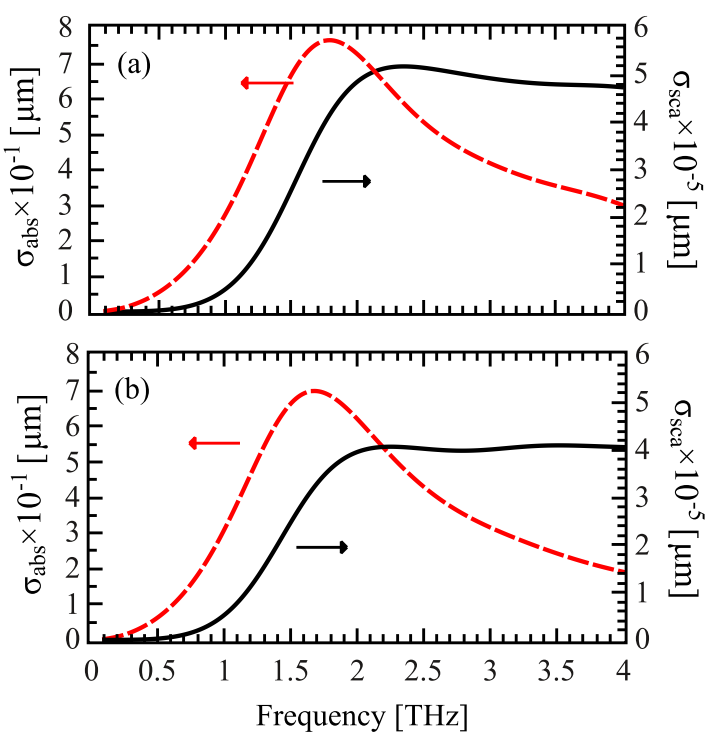

FIGURE 2. Scattering (solid black line) and absorption (dashed red line) cross sections per unit width of an infinitely wide graphene stripe with length $L=5 \mu \mathrm{m}$. Results of the Fabry-Perot model (a) are compared with the direct numerical simulations (b).

An infinite graphene layer supports transversemagnetic (TM) surface plasmon polarity (SPP) waves with the effective mode index given by [9]

$$
n_{\mathrm{eff}}=\sqrt{1-4 \frac{\mu_{0}}{\varepsilon_{0}} \frac{1}{\sigma^{2}}} .
$$

While SPP modes are not supported by free space, a termination of the graphene layer acts as a mirror and a Fabry-Perot (FP) type resonator for SPP modes can be realized in a graphene stripe of finite length. The coupling of the incident radiation with the corresponding SPP FP modes leads to the resonances in the extinction cross section of the graphene stripe shown in figure 1 .

The FP resonance condition is given by

$$
m \frac{1}{2} \frac{\lambda_{m}}{n_{\mathrm{eff}}}=L+2 \delta L,
$$

$m$ is an integer determining the order of the resonance, $L$ is the stripe length and $\delta L$ is a measure of the field penetration outside the stripe. This equation determines a set of $m$ resonance wavelengths $\lambda_{m}$ (frequencies $\omega_{m}$ ) corresponding to $m$ modes of the resonator. Taking into account that modes of the resonator are orthogonal, one can model those modes as a set of independent damped, driven harmonic oscillators with dipole moments

$$
\vec{\mu}_{m}=\frac{f_{m}}{\omega_{0 m}^{2}-\omega^{2}-\mathrm{i} \gamma_{m} \omega} \mathbf{E}_{0}=\alpha_{m} \mathbf{E}_{0}
$$


here $\omega_{0 m}=\sqrt{\left(\operatorname{Re} \omega_{m}\right)^{2}+\left(\operatorname{Im} \omega_{m}\right)^{2}}$ is the undamped angular frequency, $\gamma_{m}=-2 \operatorname{Im} \omega_{m}$ is the damping rate and $f_{m}$ is the oscillator strength. This model allows to calculate easily the scattering and absorption cross sections of the graphene stripe for the incident plane wave [10]

$$
\sigma_{s c a}=\frac{\omega}{\varepsilon_{0} c} \sum_{m=1}^{N} \operatorname{Im} \alpha_{m}, \sigma_{a b s}=\frac{\omega^{4}}{6 \pi \varepsilon_{0}^{2} c^{4}} \sum_{m=1}^{N}\left|\alpha_{m}\right|^{2} .
$$

In figure 2, the dipole model is compared with the direct numerical simulation (equivalent surface impedance method). A plane wave normally incident on the infinitely wide graphene stripe with length $L=5 \mu \mathrm{m}$ is considered. To model the graphene stripe with an infinite transverse dimension, periodic boundary conditions were assigned in this direction. The total effective length of the FP resonator $L_{e f f}=L+2 \delta L$ is assumed to be 6 $\mu \mathrm{m}$, where the penetration length $\delta L$ has been estimated based on direct numerical simulations. At normal incidence, a plane wave can couple effectively only with odd resonances, i.e., $f_{m}=0$ for odd $m$. For the even mode indexes, the oscillator strength has been used as a fit parameter and was chosen as $f_{1}=0.02, f_{1}=0.002$ and $f_{1}=0.0035$. As can be seen in figure 2 , the incident plane wave predominantly couples into the first SPP resonant mode. Moreover, absorption dominates over scattering by four orders of magnitude. A very good agreement with numerical simulation can be also observed.

The FP model can be used for a fast estimation of the spectral position of graphene-based antenna resonances. In figure 3, the position of the first resonance of the infinitely wide graphene stripe as a function of its length, as obtained from the FP model, is compared with numerical simulation using the equivalent surface impedance model. The penetration length $\delta L=0.5 \mu \mathrm{m}$ was used in the FP model. Again, the results of the numerical simulations show a very good agreement with the FP model.

A realistic patch nano-antenna, however, will have a finite width. Figure 4 shows the extinction cross section of a graphene-based antenna as a function of its width, calculated using equivalent surface impedance model. The antenna length is $L=5 \mu \mathrm{m}$. One can see that the antenna resonant frequency is reduced as the antenna becomes narrower. These results suggest that, by adjusting the antenna width and length, one can tune the antenna operation frequency in a wide spectral range.

To conclude, we have analyzed the scattering, absorption and extinction cross sections of a free-standing patch of graphene, which is a simple model of a graphenebased nano-antenna. Simulations demonstrate that a graphene-based antenna with dimensions of a few $\mu \mathrm{m}$ resonates in the terahertz band, consistently with theoretical predictions [4]. A simple FP model of the resonant electromagnetic wave interaction with the graphene antenna has been proposed and validated. Graphene-based

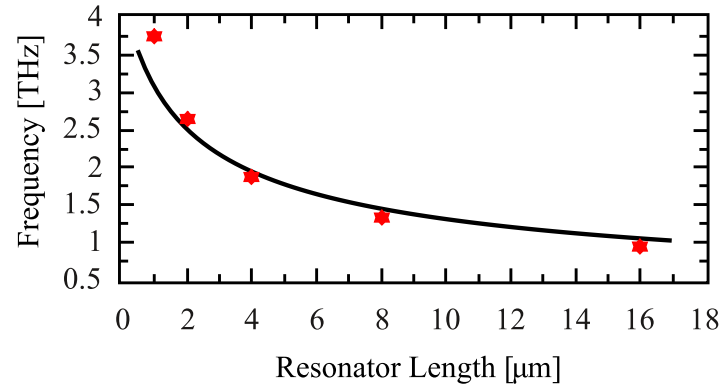

FIGURE 3. Dependence of the first resonance of the infinitely wide graphene stripe as a function of its length. Solid line is as calculated using the Fabry-Perot model, stars are resonance frequencies obtained from direct numerical simulation.

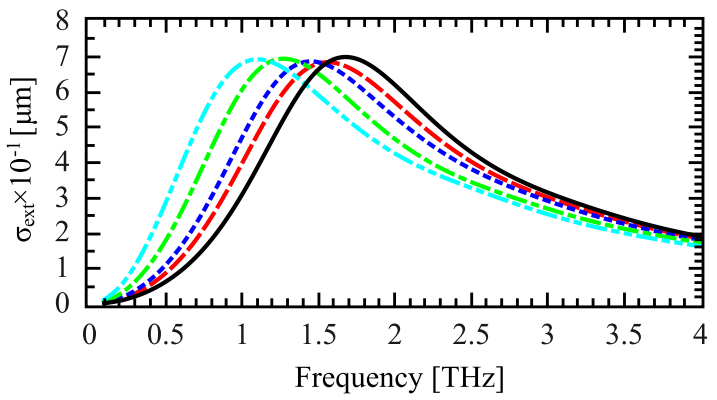

FIGURE 4. Dependence of the first resonance of the finite size graphene patch nano-antenna as a function of its width. Extinction cross section normalized to antenna width is shown. The antenna length is $L=5 \mu \mathrm{m}$. Plots correspond to infinite, 10 $\mu \mathrm{m}, 5 \mu \mathrm{m}, 2 \mu \mathrm{m}$ and $1 \mu \mathrm{m}$ wide patches (right to left).

nano-antennas are envisaged to allow nano-devices to transmit and receive information, by means of grapheneenabled wireless communications.

This work has been partially supported by the FPU grant of the Spanish Ministry of Education.

\section{REFERENCES}

1. A.K. Geim, and K.S. Novoselov, Nature Materials 6 , 183-91 (2007)

2. F. Schwierz, Nature Nanotechnology, (2010)

3. X. Wang, L. Zhi, and K. Müllen, Nano Letters 8 1, 323-327.

4. J.M. Jornet, and I.F. Akyildiz, European Conference on Antennas and Propagation, Barcelona (2010)

5. I.F. Akyildiz, and J.M. Jornet, Nano Communication Networks, 1, 3-19 (2010)

6. L. Falkovsky, and S. Pershoguba, Phys. Rev. B 76, 1-4 (2007)

7. G. W. Hanson, IEEE Trans. Ant. Prop. 56, 747-757 (2008)

8. EM Software and Systems, FEKO, www.feko.info

9. A. Vakil, and N. Engheta, Science 332, 1291 (2011)

10. L. Novotny, and B. Hecht, Principles Of Nano-Optics (Cambridge University Press, Cambridge, 2006) 